\title{
Enterococcus faecium 137v como fator de proteção em modelo animal para câncer colorretal
}

\author{
Enterococcus faecium $137 \mathrm{v}$ as a protective factor in an animal model for colorectal cancer \\ Enterococcus faecium $137 \mathrm{v}$ como factor de protección en modelo animal para el cáncer colorrectal
}

Recebido: 22/04/2021 | Revisado: 05/05/2021 | Aceito: 07/05/2021 | Publicado: 21/05/2021

Priscilla Régia de Andrade Calaça

ORCID: https://orcid.org/0000-0001-9092-6832

Universidade Federal Rural de Pernambuco, Brasil

E-mail: priscilla.acalaca@ufrpe.br

Dayane da Silva Santos

ORCID: https://orcid.org/0000-0001-8324-7858

Universidade Federal de Pernambuco, Brasil

E-mail: santos.dhay08@gmail.com

Janaína Ferreira da Silva

ORCID: https://orcid.org/0000-0002-8051-5834

Universidade Federal Rural de Pernambuco

E-mail: janainaferreiravet@gmail.com

Ana Beatriz Lins Aragão

ORCID: https://orcid.org/0000-0002-0440-8535

Universidade Federal Rural de Pernambuco, Brasil E-mail: beatrizaragao27@gmail.com

Ismaela Maria Ferreira de Melo

ORCID: https://orcid.org/0000-0002-4150-1923

Universidade Federal Rural de Pernambuco, Brasil E-mail: ismaelamelo@yahoo.com.br

Elayne Cristine Soares da Silva

ORCID: https://orcid.org/ 0000-0002-0257-6527

Universidade Federal Rural de Pernambuco, Brasil

E-mail: elayne.silva@ufrpe.br

Ana Lúcia Figueiredo Porto

ORCID: https://orcid.org/0000-0001-5561-5158

Universidade Federal Rural de Pernambuco, Brasil

E-mail: analuporto@yahoo.com.br

Maria Taciana Cavalcanti Vieira Soares

ORCID: https://orcid.org/0000-0001-9573-6296

Universidade Federal Rural de Pernambuco, Brasil E-mail: maria.vsoares@ufrpe.br

\begin{abstract}
Resumo
Enterococcus faecium é um importante gênero bactérias ácido láticas probióticas que forma parte integrante da microbiota humana saudável. Entretanto, poucos estudos relacionam a capacidade da Enterococcus faecium de origem alimentar para prevenção do câncer colorretal, considerado uma das principais causas de mortalidade nos países ocidentais, estando entre os três cânceres mais incidentes. O nosso estudo se propõe em utilizar uma nova abordagem para avaliar o potencial da cepa Enterococcus faecium 137v - EF137v de queijo de Coalho artesanal no seu desempenho para prevenção e retardo na progressão do câncer colorretal em modelo animal. Para este fim avaliamos, através da ultrassonografia abdominal, os efeitos da administração oral da EF137v sobre o desenvolvimento do câncer colorretal induzido por 1,2-dimetilhidrazina - DMH em diversos grupos objetivando uma análise sistêmica na saúde dos ratos Wistar, bem como observações de possíveis alterações no peso e níveis séricos desses animais. Os resultados obtidos foram importantes para o retardo na progressão tumoral promovido por EF137v em ratos que fizeram uso do probiótico de modo isolado antes (preventivo) e após (curativo) a indução do câncer. Os ratos apresentaram peso corporal estável, sem mudanças bruscas no decorrer do experimento. Níveis séricos de triglicerídeos apresentaram-se altos após o câncer na maioria dos grupos tratados. Uma discussão sobre possíveis alterações metabólicas foi iniciada, bem como a função protetora da EF137v ao câncer colorretal. O probiótico EF137v mostrou-se forte candidato como agente preventivo para o desenvolvimento do câncer e o procedimento ultrassonográfico uma ferramenta adicional para o acompanhar alterações importantes no intestino.
\end{abstract}

Palavras-chave: Câncer colorretal; Enterococcus faecium; Probiótico; Ratas Wistar; Ultrassonografia. 


\begin{abstract}
Enterococcus faecium is an important genus of probiotic acid lactic bacteria that forms an integral part of the healthy human microbiota. However, few studies relate the ability of food-borne Enterococcus faecium to prevent colorectal cancer, considered one of the main causes of mortality in Western countries, being among the three most incident cancers. Our study proposes to use a new approach to evaluate the potential of the Enterococcus faecium 137v EF137v strain of artisanal "Coalho" cheese in its performance for prevention and delay in the progression of colorectal cancer in an animal model. To this end, we evaluated, through abdominal ultrasound, the effects of oral administration of EF137v on the development of colorectal cancer induced by 1,2-dimethylhydrazine - DMH in several groups aiming at a systemic analysis on the health of Wistar rats, as well as observations of possible changes in the weight and serum levels of these animals. The results obtained were important for the delay in tumor progression promoted by EF137v in rats that used the probiotic in isolation before (preventive) and after (dressing) the cancer induction. The rats had a stable body weight, with no sudden changes during the experiment. Serum triglyceride levels were high after cancer in most treated groups. A discussion on possible metabolic changes was initiated, as well as the protective function of EF137v against colorectal cancer. The probiotic EF137v proved to be a strong candidate as a preventive agent for the development of cancer and the ultrasound procedure is an additional tool to accompany important changes in the intestine.
\end{abstract}

Keywords: Colorectal cancer; Enterococcus faecium; Probiotic; Wistar rats; Ultrasonography.

\title{
Resumen
}

Enterococcus faecium es un género importante de bactérias ácido láctica probióticas que forma parte integral de la microbiota humana sana. Sin embargo, pocos estudios relacionan la capacidad del Enterococcus faecium transmitido por los alimentos para prevenir el cáncer colorrectal, considerado una de las principales causas de mortalidad en los países occidentales, estando entre los tres cánceres más incidentes. Nuestro estudio propone utilizar un nuevo enfoque para evaluar el potencial de la cepa Enterococcus faecium 137v - EF137v de queso de "Coalho" artesanal en su desempeño para prevenir y retrasar la progresión del cáncer colorrectal en un modelo animal. Para ello, evaluamos, mediante ecografía abdominal, los efectos de la administración oral de EF137v sobre el desarrollo de cáncer colorrectal inducido por 1,2-dimetilhidrazina - DMH en varios grupos con el objetivo de realizar un análisis sistémico sobre la salud de ratas Wistar, así como observaciones de posibles cambios en el peso y los niveles séricos de estos animales. Los resultados obtenidos fueron importantes para el retraso en la progresión tumoral promovido por EF137v en ratas que usaron el probiótico de forma aislada antes (preventivo) y después (vendaje) de la inducción del cáncer. Las ratas tenían un peso corporal estable, sin cambios bruscos durante el experimento. Los niveles séricos de triglicéridos fueron altos después del cáncer en la mayoría de los grupos tratados. Se inició una discusión sobre posibles cambios metabólicos, así como la función protectora de EF137v contra el cáncer colorrectal. El probiótico EF137v demostró ser un fuerte candidato como agente preventivo para el desarrollo de cáncer y el procedimiento de ultrasonido es una herramienta adicional para acompañar cambios importantes en el intestino.

Palabras clave: Cáncer colorrectal; Enterococcus faecium; Probiótico; Ratas Wistar; Ultrassom.

\section{Introdução}

O câncer colorretal é considerado uma das principais causas de mortalidade nos países ocidentais, estando entre os três cânceres mais incidentes em homens e mulheres (Jaskulski, et al., 2020). Já há tempo que os tratamentos disponíveis para o câncer incluem quimioterapia e cirurgia, estes contribuem enormemente para a perda de qualidade de vida. Neste sentido, há uma grande necessidade de um tratamento alternativo por intervenção nutricional que inclua agentes com potenciais ações quimiopreventivas (Urbanska, et al., 2009).

Evidências indicam que o consumo de bactérias ácido láticas - BAL probióticas melhora a função intestinal, mantendo a permeabilidade paracelular, aumentando a camada mucosa física, estimulando o sistema imunológico e modulando a composição e atividade da microbiota residente (Khan, et al., 2017). BAL probióticas são principalmente mostradas para impedir o crescimento de células cancerosas indiretamente por meio do aumento da imunidade adaptativa pela indução de células T citotóxicas (Lenoir, et al., 2016), células natural killer - NK ou citocinas (Sharma, et al., 2018).

Enterococcus faecium (E. faecium) é um importante gênero BAL que forma parte integrante da microbiota humana saudável. O gênero Enterococcus também é encontrado na microbiota de fontes alimentares diversas, como queijo de Coalho, por exemplo. Em animais, os probióticos de E. faecium são usados principalmente para tratar ou prevenir diarreia, para facilitar a estimulação imunológica ou para melhorar o crescimento (Wu, et al., 2019). Poucos estudos demonstraram as propriedades de proteção ao câncer de cepas de Enterococcus de origem alimentar in vitro (Thirabunyanon, et al., 2013; Naomi, et al., 
2015). Raros trabalhos in vitro com Enterococcus faecium de origem alimentar são identificados para avaliar a capacidade de anticâncer (Thirabunyanon, et al., 2009). Atualmente, o nosso estudo torna-se pioneiro por avaliar in vivo a ação protetora e adjuvante na terapia contra o câncer colorretal da Enterococcus faecium de origem alimentar.

Muitos estudos confirmam o envolvimento da microbiota exógena no aparecimento do câncer colorretal. Isso torna razoável pensar que a mudança da microbiota intestinal pode influenciar o desenvolvimento do tumor. A 1,2-dimetil-hidrazina (DMH) é um potente carcinógeno que pode induzir câncer colorretal em animais experimentais (Kumar, et al., 2012). Os humanos são expostos ao DMH e outras hidrazinas por meio do ambiente e da dieta. O DMH é metabolizado no fígado, resultando na produção de íon diazônio eletrofílico, que é conhecido por provocar estresse oxidativo (Desrouillères, et al., 2015). Segundo Kumar, et al. (2012) câncer colorretal experimental provocado pela administração de DMH em ratos apresenta estágios múltiplos prolongado, apresentando muitas das mesmas cinéticas celulares, características histopatológicas e moleculares que simula o desenvolvimento do câncer de cólon humano.

O nosso estudo se propõe em utilizar uma nova abordagem para avaliar o potencial da BAL Enterococcus faecium 137v oriunda de queijo de Coalho artesanal do Nordeste do Brasil em seu desempenho na prevenção e retardo na progressão do câncer colorretal em modelo animal e na manutenção da saúde sistêmica.

\section{Metodologia}

Este estudo se caracteriza por sua natureza quantitativa, analítica, intervencional e experimental de acordo com Hochman, et al. (2005).

\subsection{Micro-organismo}

A cepa utilizada nesta pesquisa está cadastrada no Sistema Nacional de Gestão do Patrimônio Genético e do conhecimento tradicional associado (SISGEN) $n^{\circ}$ AD77AD8. É oriunda do queijo de Coalho artesanal de Pernambuco e foi classificada como Enterococcus faecium 137v não patogênica e com perfil probiótico baseada por filogenia (Santos, et al., 2020). A cepa foi cultivada (10\% de inúculo) em caldo de Man, Rogosa e Sharpe (MRS - HiMedia Laboratories Pvt, Ltd. Mumbai, India) a $37^{\circ} \mathrm{C} / 24 \mathrm{~h}$. O cultivo foi centrifugado a $6.500 \mathrm{rpm} / 20$ minutos. O sobrenadante foi cuidadosamente descartado e as células bacterianas retidas pela centrifugação foram ressuspendidas em água ultrapura e padronizadas a uma concentração de $10^{10}$ unidades formadoras de colônia - UFC. A E. faecium $137 \mathrm{v}$ foi armazenada em frascos contendo $10 \%$ de glicerol e submetida ao congelamento gradual até que alcançasse $-80^{\circ} \mathrm{C}$. Após processo de congelamento a amostra foi liofilizada. O material obtido da liofilização foi armazenado sob congelamento para fins de análise experimental.

\subsection{Animais}

Quarenta e dois ratos Wistar (Rattus norvergicus, variedade albinus, Rodentia) fêmeas com cinco semanas de idade e pesando em média 112,62 £ 6,44 g foram obtidos do Biotério de Criação e Experimentação do Departamento de Morfologia e Fisiologia Animal (Universidade Federal Rural de Pernambuco, DMFA/UFRPE) e alojados em gaiolas de arame inoxidável. A temperatura e a umidade relativa foram mantidas em $22^{\circ} \mathrm{C}$ e $55 \%$, respectivamente. Os ciclos de claro-escuro duravam 12 horas cada. Todos os animais tiveram livre acesso à água e pellets comerciais de ração para ratos fornecidos pelo Biotério. Todos os cuidados e tratamentos com os animais experimentais seguiram as diretrizes estabelecidas pelo Comitê de Ética de Uso Animal (CEUA/UFRPE) sob a licença: 001/2018.

\subsection{Desenho experimental}

O ratos foram submetidos a um período de aclimatação de uma semana. Após esse período, eles foram divididos 
aleatoriamente em 7 grupos de 6 animais, cada $(n=42)$. Todos os ratos foram alimentados com dieta comercial fornecida pelo Biotério.

O Grupo $1(\mathrm{GN})$ serviu como controle negativo e recebeu $0,85 \%$ de solução salina $(\mathrm{NaCl} 0,85 \%)$ por gavagem (intragástrica i.g.) diariamente. Os ratos do grupo 2 (GP) serviram como controle positivo para o câncer e receberam 1,2Dimetilhidrazina-DMH (Kumar, et al., 2012; Desrouillères, et al., 2015) dissolvido em $\mathrm{NaCl}$ 0,85\% (30 mg/kg por via subcutânea - s.c.) uma vez por semana durante seis semanas. O Grupo 3 (G3) serviu como controle para o probiótico recebendo Enterococcus faecium 137v - EF137v por gavagem diariamente. Os grupos 4 a 7 (G4, G5, G6 e G7) receberam o EF137v (i.g.) e DMH (s.c.). O G4 e G7 receberam sulfato de vincristina - VCR (1 mg/ml de $\mathrm{NaCl}$ 0,85\%) e 0,3 $\mathrm{ml}$ desta solução foi administrada por via intravenosa (i.v.) semanalmente após indução do câncer. O G4 e G5 fizeram o uso de EF137v durante toda a fase experimental (Fase 1: antes da indução do câncer; Fase 2: durante a indução do câncer; e Fase 3: após indução do câncer). O G6 e G7 iniciaram o consumo do EF137v na Fase 3. A dose probiótica foi suplementada com 1,0 ml de Enterococcus faecium $137 \mathrm{v}$ - EF137v a uma concentração de $10^{10} \mathrm{UFC} / \mathrm{ml}(0,0115 \mathrm{~g}$ EF137v liofilizada solubilizada em $\mathrm{NaCl}$ $0,85 \%)$.

Os animais foram monitorados diariamente quanto à saúde geral e os pesos corporais foram registrados periodicamente durante o estudo. Após 21 semanas de período experimental, os ratos foram sacrificados com administração, por via intramuscular de sobredosagem de cetamina $300 \mathrm{mg} / \mathrm{kg}$ + xilazina $30 \mathrm{mg} / \mathrm{kg}$, após confirmação da perda de consciência, realizou-se exsanguinação por punção cardíaca e morte. Todo o segmento do intestino foi coletado para análise, bem como o fígado, rim, estômago, baço, pâncreas, pulmão, ovário, útero e coração e armazenados em solução de álcool a 70\% para análises histológicas. O desenho experimental é descrito na Figura 1. Os ratos Wistar albinos fêmeas foram empregadas neste estudo, por serem utilizadas em estudos semelhantes anteriores (Gamallat, et al., 2016; Lenoir, et al., 2016). 
Figura 1. Representação do desenho experimental.

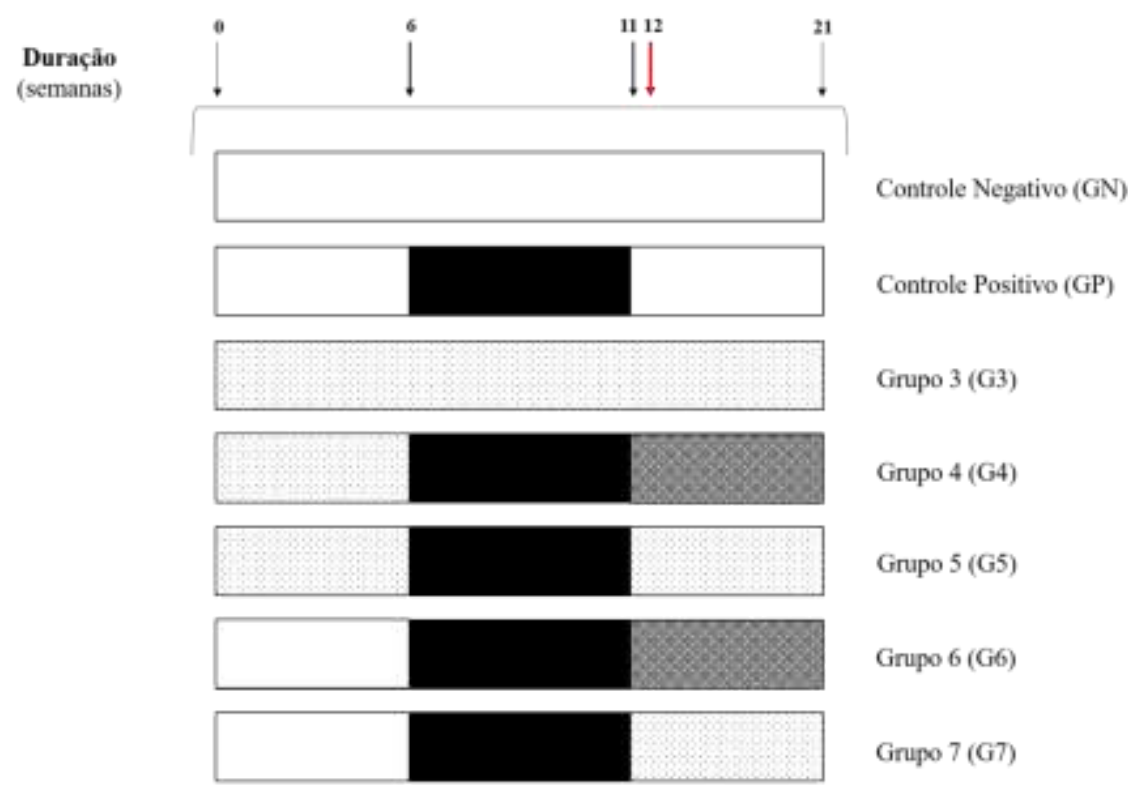

Indica a injeção de VCR

(1 mg/ml de $\mathrm{NaCl} 0,85 \%$, i.v.)

Dieta normal $+\mathrm{NaCl} 0,85 \%$

Injeçăo de DMH

( $30 \mathrm{mg} / \mathrm{kg}, 5 . \mathrm{c}$.)

Dieta normal $+\mathrm{EF} 137 \mathrm{v}$

Dieta normal + EF137v + VCR

Fonte: Autores.

\subsection{Ultrassonografia abdominal}

Os ratos foram submetidos a avaliação ultrassonográfica (Sonosite, M-turbo, 6 a $13 \mathrm{MHz}$ transdutor linear) para avaliar os parâmetros anatomopatológicos do intestino nas fases 1, 2 e 3. As análises por ultrassonografia incluíram a observação da ecogenicidade intestinal, hepática e renal (EcoI/EcoH/Eco-R) e a aferição do diâmetro transverso da parede intestinal (DT).

\subsection{Análise Bioquímica}

O colesterol total e triglicerídeos foram determinados nas amostras de soro coletadas dos ratos Wistar nas fases 1 e 3 deste trabalho utilizando kit comercial (Diagnóstica InVitro®, Brasil).

\subsection{Análise estatística}

Os dados quantitativos foram expressos como desvio padrão médio. As variáveis foram analisadas usando o ANOVA seguido pelo teste Bonferroni e Tukey para comparações entre médias ou análise de variância unilateral e comparações post hoc de Shapiro-Wilk, bem como metanálise nos softwares Jamovi 1.6 (para a maioria dos testes em estudo). O critério de significância estatística foi estabelecido em $\mathrm{p}<0.05$ para cada ponto final. 


\section{Resultados}

\subsection{Ganho de peso corporal}

A Figura 2 mostra um aumento constante no peso corporal dos animais de todos os grupos testados $(112,62 \pm 6,44$ a $187,37 \pm 3,72 \mathrm{~g}$ ao longo de 21 semanas). Foi observado um peso corporal estável nos animais do grupo G5 que receberam o probiótico antes da indução do câncer e continuaram o seu uso após indução: 170,67 $\pm 14,57$ a 181,00 $\pm 17,13 \mathrm{~g}$ (fase 2) e $183,60 \pm 18,15$ a $203,60 \pm 17,36 \mathrm{~g}$ (fase 3).

Após 6 semanas de injeção do cancerígeno DMH os grupos G6 e G7 apresentaram um rápido declínio no peso corporal (201,00 $\pm 13,66$ para 197,80 \pm 18,01 e 180,50 \pm 15,23 para 179,50 $\pm 14,71 \mathrm{~g}$, respectivamente) em comparação aos demais animais tratados, no entanto, ao longo do tratamento, o peso dos animais foi recuperado. A Figura 3 mostra que os ratos tiveram seus pesos estabilizados, sem aumento significativo durante a fase 2 para a fase $3(p \geq 0,05)$ quando comparados com a fase 1 seguida das fases 2 e 3 ( $\mathrm{p}=0,001$ ).

Figura 2. Histograma e densidade estatística $(\mathrm{p} \leq 0,05)$. Aumento significativo de peso médio $(\mathrm{p}=0,009)$ em ratos Wistar para os grupos testados durante as fases $1,2 \mathrm{e} 3 \mathrm{em} 21$ semanas.
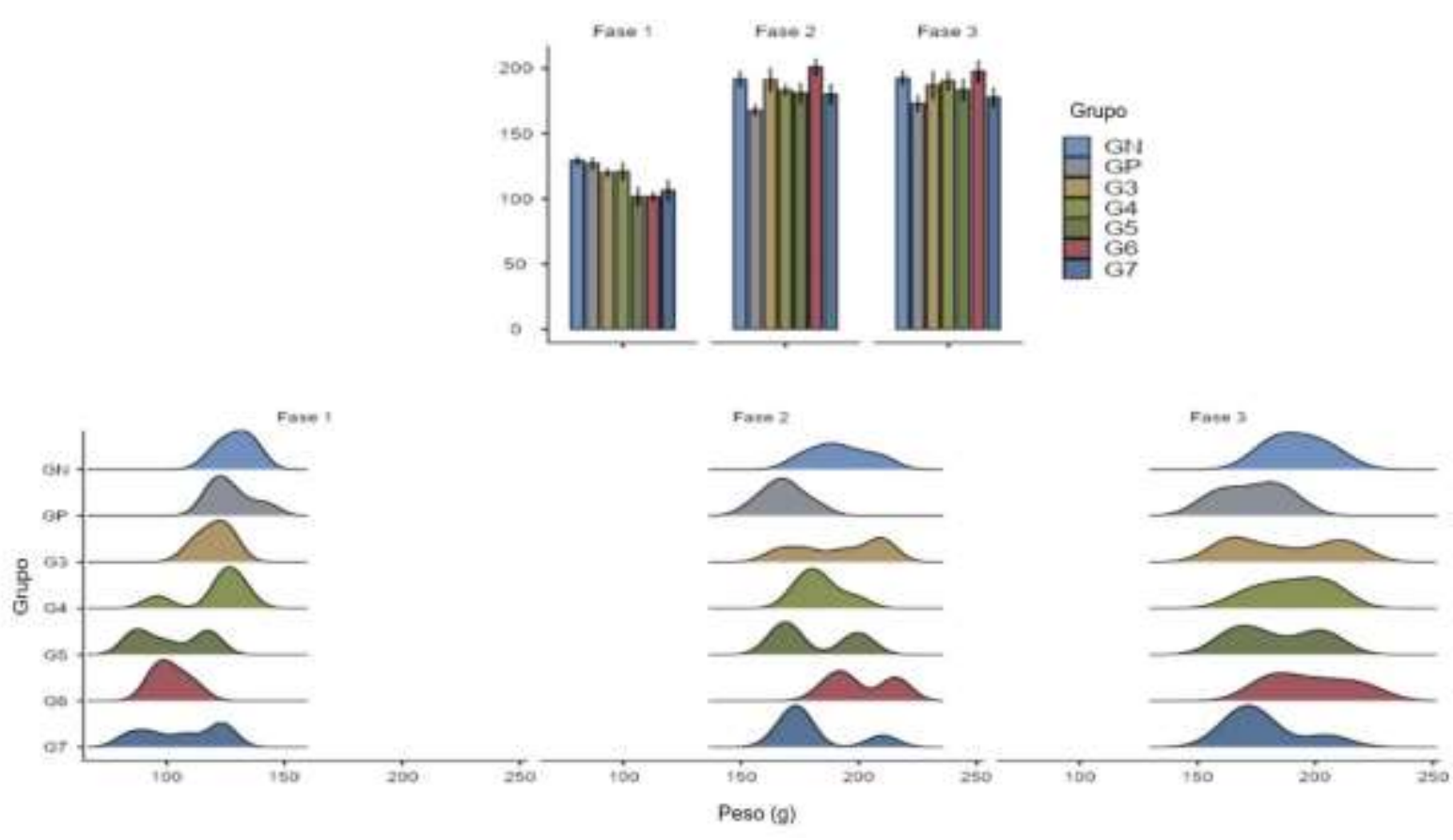

Fonte: Autores em software estatístico Jamovi (versão 1.6). 
Figura 3. Comparação ANOVA - Post Hoc por fase comparando o peso corporal médio de ratos em cada grupo ( $\mathrm{p} \leq 0,05)$.

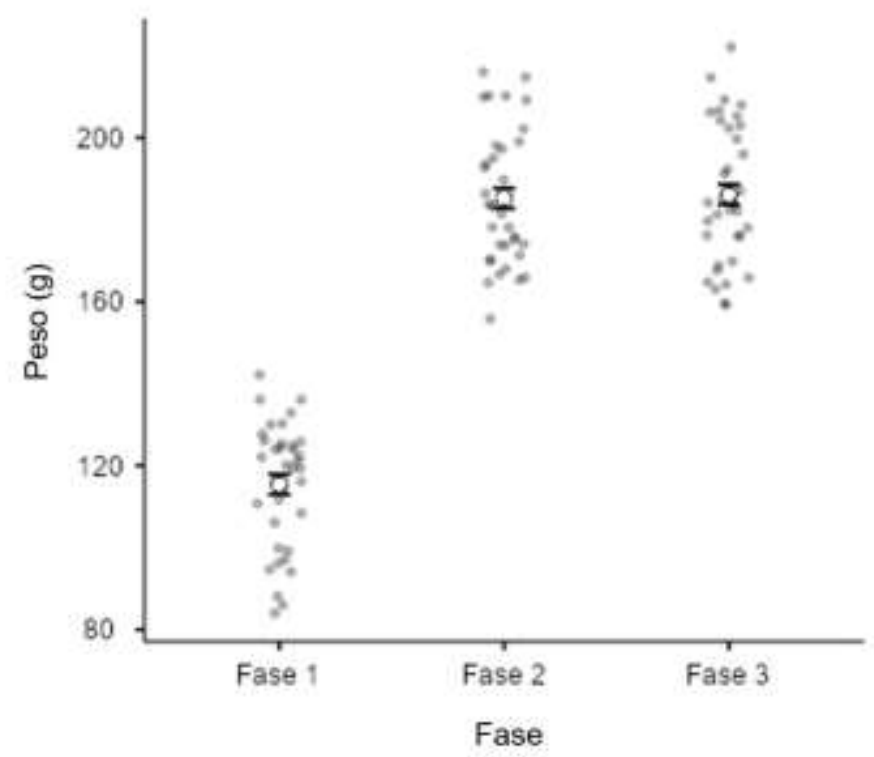

Nota. As comparações são baseadas em médias marginais estimadas. Fonte: Autores em software estatístico Jamovi (versão 1.6).

\subsection{Ultrassonografia abdominal}

Durante a fase 1 não foram observadas alterações nos rins, fígado, baço, bexiga e intestino para todos os grupos avaliados. A Figura 4 mostra o diâmetro da parede intestinal em que o grupo G4 (14 $\pm 0,1 \mathrm{~mm})$ estava na terceira semana de administração do EF137v. O grupo G7 $(15,5 \pm 0,7 \mathrm{~mm})$ que neste momento não consumia o probiótico e GP (10 $\pm 1,41 \mathrm{~mm})$ foram avaliados para observações nas demais fases quanto ao acompanhamento das condições do intestino frente ao câncer. 
Figura 4. Imagem ultrassonográfica do diâmetro transverso da parede intestinal (DT) dos grupos GN (A), GP (B), G4 (C) e G7 (D) na fase 1 experimental.
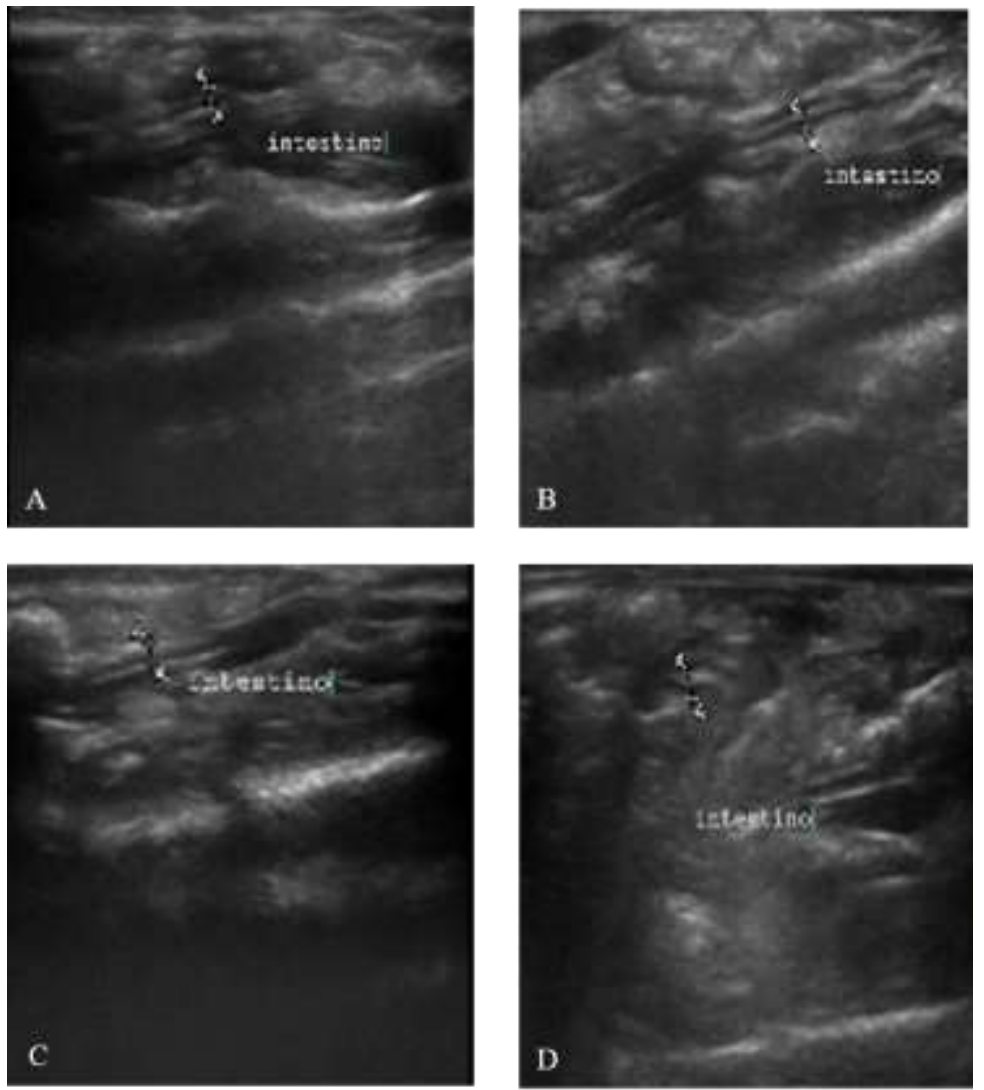

Fonte: Autores.

$\mathrm{Na}$ fase 2 do experimento foram observadas algumas mudanças no estado de saúde dos ratos e detectadas através da ultrassonografia, como mostra a Figura 5. Três ratas do grupo G6 $(20,5 \pm 3,53 \mathrm{~mm})$ apresentaram leve aumento da ecogenicidade de mesentério, esplenomegalia e peristaltismo aumentado, embora não tenham apresentado diarreia. Um animal do grupo G5 (20,5 $\pm 0,7 \mathrm{~mm})$ apresentou esplenomegalia. 
Figura 5. Imagem ultrassonográfica do diâmetro transverso da parede intestinal (DT) na fase 2 dos grupos GP (A), G5 (B), G6 (C) e G7 (D) e na fase 3 dos grupos GP (E), G5 (F), G6 (G) e G7 (H).
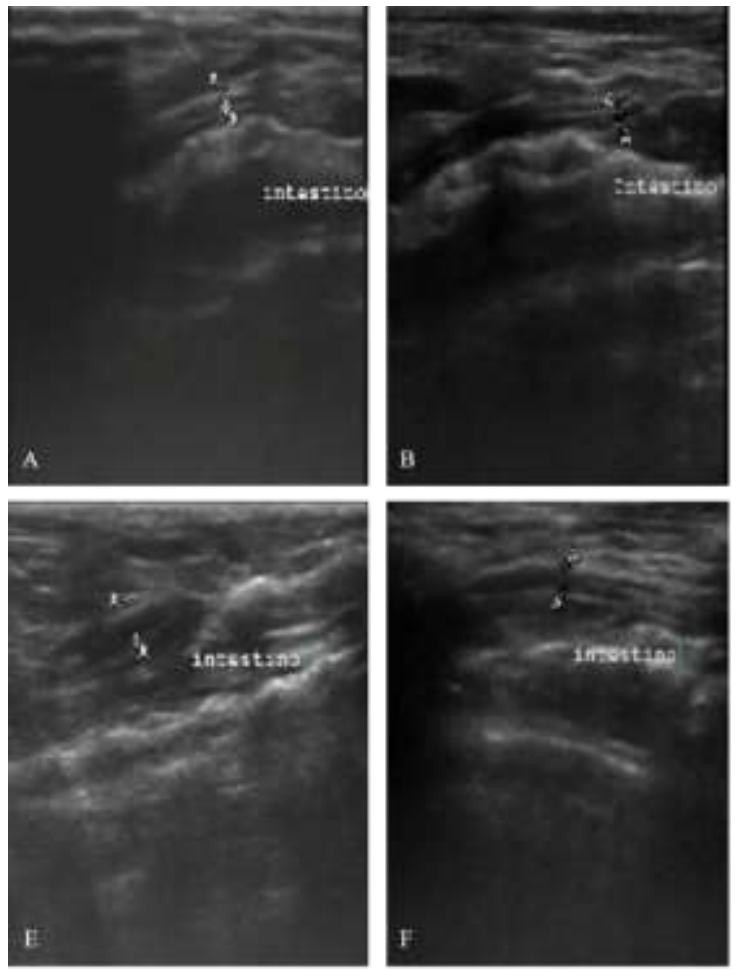
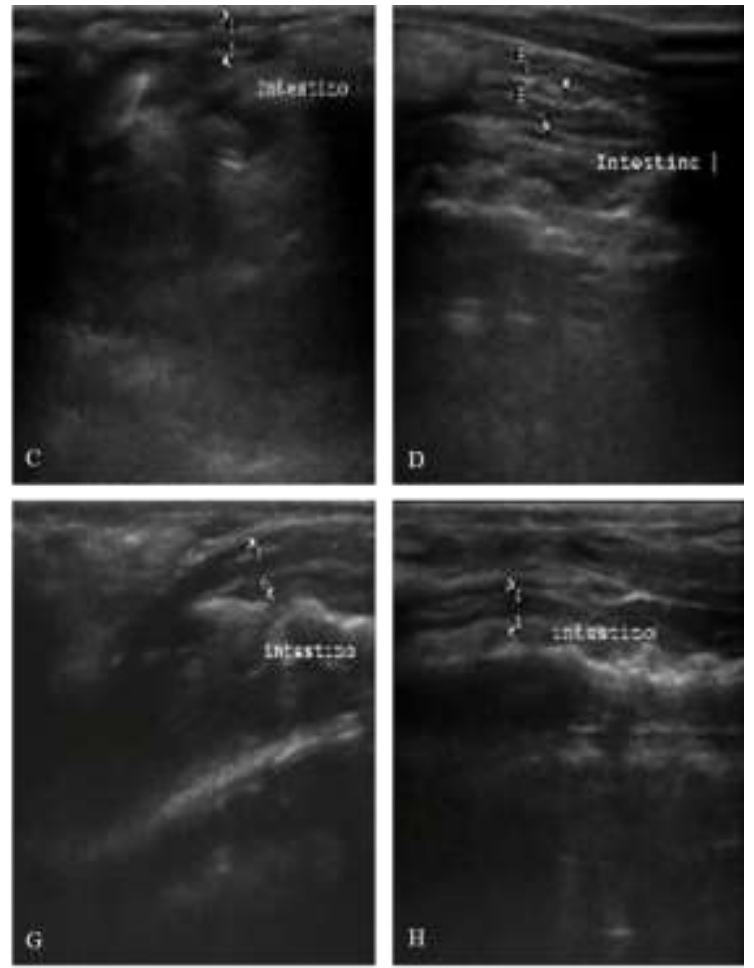

Fonte: Autores.

Um aumento DT foi observado no grupo GP durante o experimento $10 \pm 1,41 \mathrm{~mm}, 19,5 \pm 0,7 \mathrm{~mm}$ e $28,5 \pm 2,12 \mathrm{~mm}$ fase 1 , fase 2 e fase 3 , respectivamente ( $\mathrm{p}=0,01)$, quando comparado ao controle negativo GN (11,5 $\pm 2,12 \mathrm{~mm})$. Os grupos G5 e G6 apresentaram aumento da parede intestinal na fase $2(17,5 \pm 3,54$ e 20,5 \pm 3,53 mm) com leve aumento na fase 3 G5 20,5 $\pm 0,7(\mathrm{p}=0,05)$ e G6 20,5 $\pm 0,7 \mathrm{~mm}(\mathrm{p}=0,02)$, quando comparados aos grupos G4 19,5 $\pm 0,7 \mathrm{~mm}$ (fase 2) e 26,5 $\pm 0,7 \mathrm{~mm}($ fase 3) e G7 23,5 \pm 0,7 mm (fase 2) e $24 \pm 1,41$ (fase 3), com aumento significativo DP do G4 (p=0,005) e G7 (p=0,003) quando comparados aos grupos que não utilizavam o EF137v associado ao VCR e ao GN, podendo ser observadas na Figura 6. O grupo G3 que fez uso de probiótico durante toda a fase experimental, não apresentou aumento DP quando comparado ao controle GN ( $\mathrm{p} \geq 0,05)$, como explicitado na Tabela 1 .

Ao realizar o teste de poder ( $t$-test) para os resultados obtidos em relação a sensibilidade da análise sobre o número de indivíduos avaliados, obteve-se um poder de $\mathrm{d}=0,695$, em que a probabilidade de detectar o efeito foi de $80-95 \%$ como mostra a Figura 7. Neste estudo por ultrassonografia a intenção é de detectar, de forma confiável, efeitos maiores da EF137v sobre o intestino frente ao desenvolvimento do câncer, logo, tamanhos de amostra menores foram necessários. 
Figura 6. BoxPlot de descrição estatística $(\mathrm{p} \leq 0,05)$. Aumento moderado de $\mathrm{G} 5$ e $\mathrm{G} 6$ ( $\mathrm{p}=0,05$ e $\mathrm{p}=0,02$, respectivamente). Forte aumento na parede do intestino nos grupos G4, G7 e GP ( $\mathrm{p}=0,005, \mathrm{p}=0,003$ e p=0,01, respectivamente) quando comparados ao GN. O grupo G3 não apresentou diferença estatística em relação ao controlo negativo.
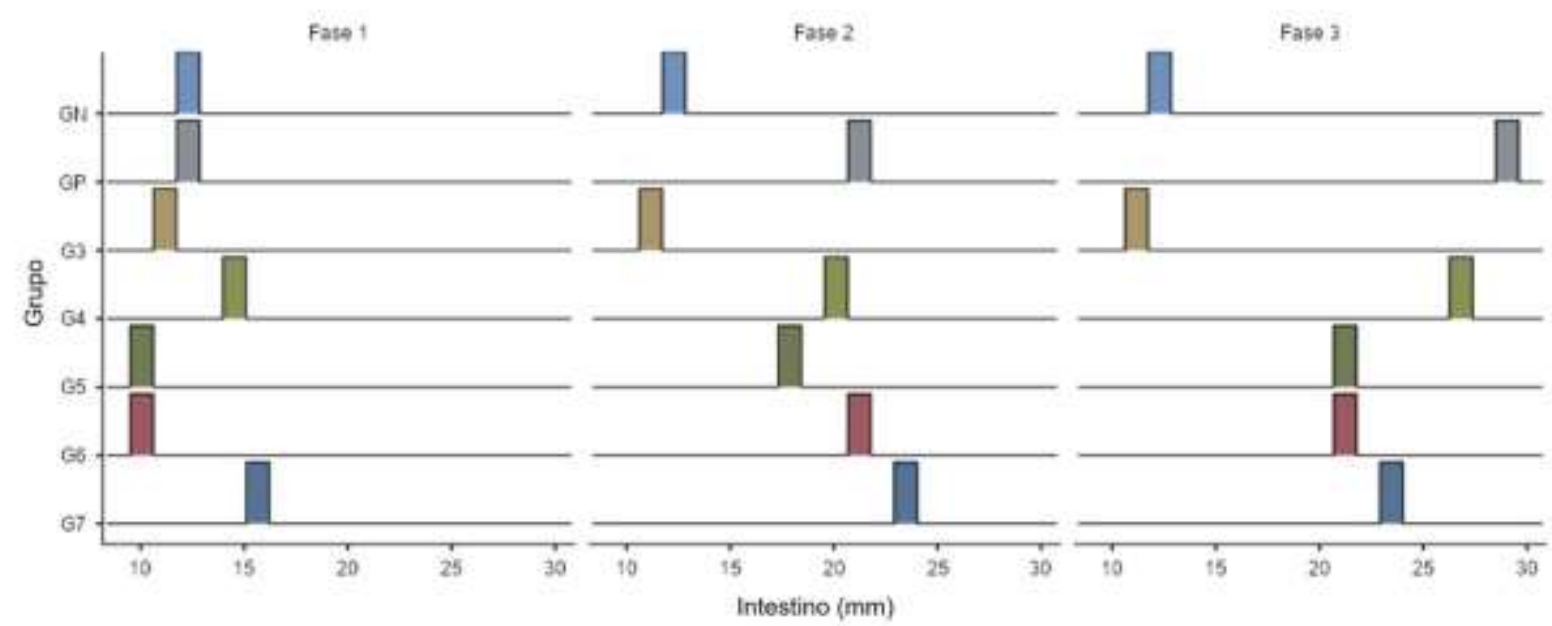

Fonte: Autores.

Tabela 1. Aumento moderado de G5 e G6 ( $\mathrm{p}=0,05$ e p=0,02, respectivamente). Forte aumento na parede do intestino nos grupos G4, G7 e GP ( $\mathrm{p}=0,005$, $\mathrm{p}=0,003$ e $\mathrm{p}=0,01$, respectivamente) quando comparados ao GN. O grupo G3 não apresentou diferença estatística em relação ao controlo negativo (ANOVA, seguido de teste de regressão logística ordinal, $\mathrm{p} \leq 0,05$ ).

\section{Indicadores $\quad \mathbf{p} \leq \mathbf{0 , 0 5} \quad$ Odds ratio}

\section{Grupo}

$\begin{array}{ccc}\text { G3 - GN } & 0.802 & 1.36344 \\ \text { G4 - GN } & 0.005 & 0.00615 \\ \text { G5 - GN } & 0.052 & 0.04515 \\ \text { G6-GN } & 0.024 & 0.01778 \\ \text { G7 - GN } & 0.003 & 0.00190 \\ \text { GP - GN } & 0.011 & 0.00717\end{array}$

Intestino $(\mathbf{m m}) \quad<.001 \quad 1.85897$

Fonte: Autores em software estatístico Jamovi (versão 1.6$)$. 
Figura 7. Poder do teste (T-test/Power Contour) O gráfico de contorno de potência mostra como a sensibilidade do teste muda com o tamanho do efeito hipotético e os tamanhos da amostra do experimento. A curva preta sólida no gráfico de contorno mostra combinações de tamanho de amostra/tamanho de efeito com uma potência de 0,695. O ponto mostra o design especificado e o tamanho do efeito.

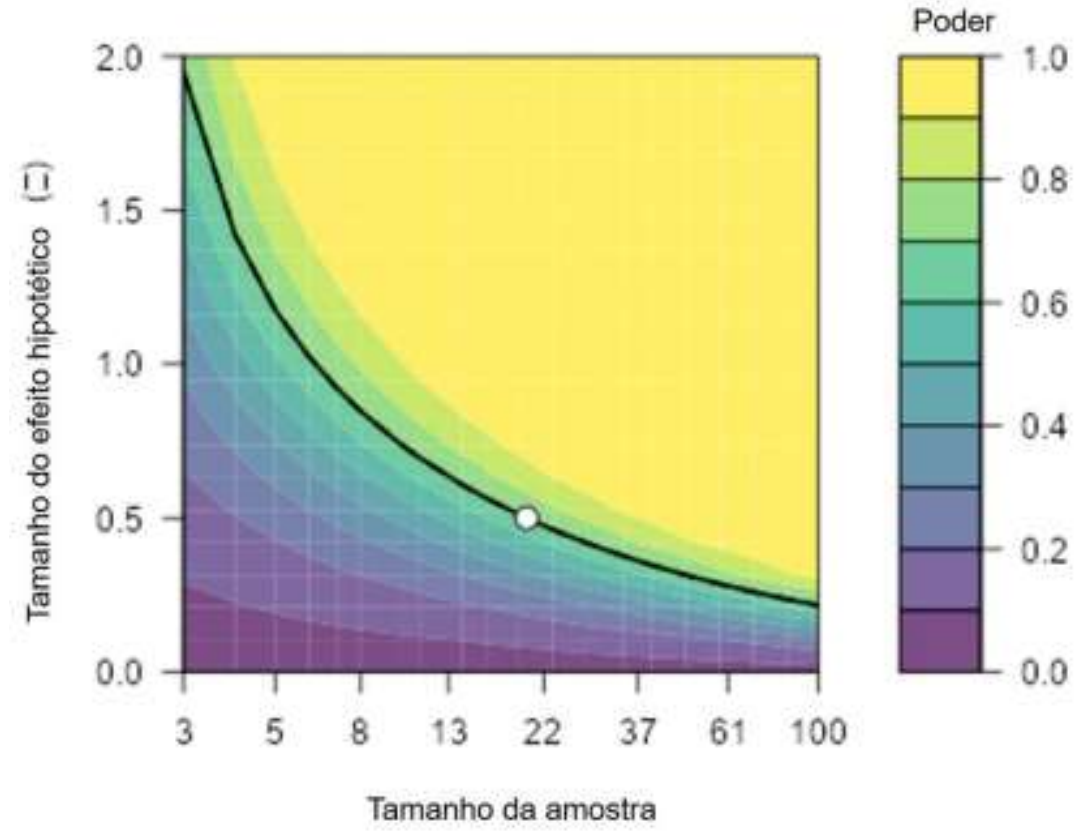

Fonte: Autores em software estatístico Jamovi (versão 1.6).

\subsection{Perfil bioquímico}

Os níveis de colesterol total de todos os grupos avaliados manteve-se em proporções desejáveis $\leq 190 \mathrm{mg} / \mathrm{dL}$ antes da indução do câncer pelo agente carcinogênico DMH, enquanto que o triglicerídeo se apresentou dentro das proporções desejáveis $\leq 175 \mathrm{mg} / \mathrm{dL}$ e limítrofe $175-199 \mathrm{mg} / \mathrm{dL}$. Um aumento considerável nos níveis de triglicerídeo para os grupos testados $(\mathrm{p}=0,02)$ ocorreu após indução do câncer, quando comparados ao grupo GN (199 \pm 44,4 mg/dL). Não houve alteração significativa para os índices de colesterol total $(\mathrm{p} \geq 0,05)$ em relação ao controle negativo em todas as etapas analisadas como mostra a Figura 8 e descrita na Tabela 2 em que as fases e grupos são comparados e avaliados quanto ao aumento dos níveis séricos apresentados. 
Figura 8. Metanálise do tamanho do efeito e variação da amostragem sobre os níveis de colesterol e triglicerídeo dos ratos, por grupo, antes e depois da indução do câncer por DMH. Forest Plot, $(\mathrm{p} \leq 5)$.
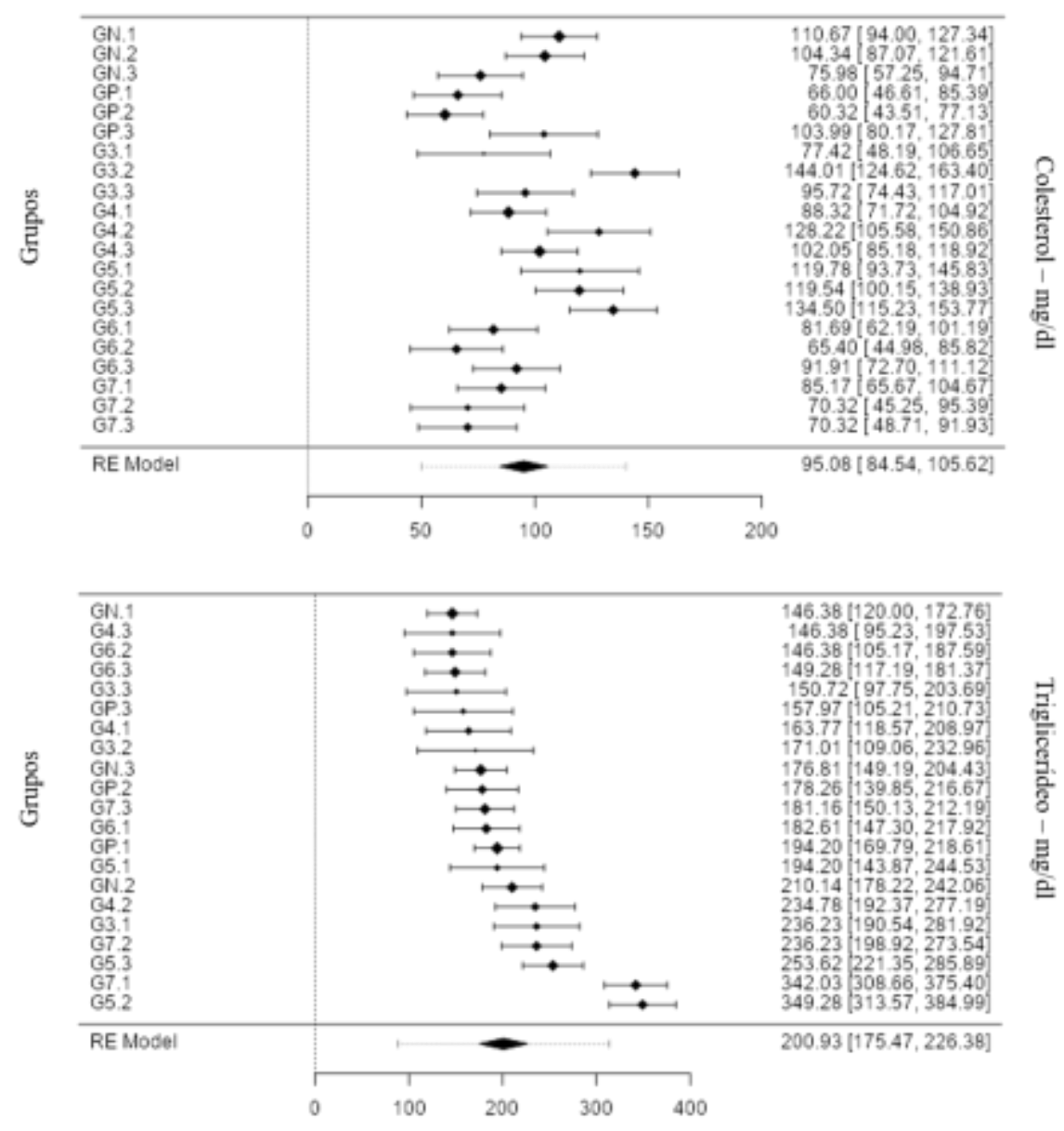

Fonte: Autores em software estatístico Jamovi (versão 1.6).

Tabela 2. Análise MANCOVA $(\mathrm{p} \leq 0,05)$ dos níveis de triglicerídeo e colesterol total antes da indução do câncer e após indução do câncer por DMH.

\begin{tabular}{llc}
\hline & \multicolumn{1}{c}{ Indicadores } & $\mathbf{p} \leq \mathbf{0 , 0 5}$ \\
\hline \multirow{2}{*}{ Fase } & Cholesterol $(\mathrm{mg} / \mathrm{dL})$ & 0.098 \\
& Triglyceride $(\mathrm{mg} / \mathrm{dL})$ & $<.001$ \\
\multirow{2}{*}{ Grupo } & Cholesterol $(\mathrm{mg} / \mathrm{dL})$ & 0.196 \\
& Triglyceride $(\mathrm{mg} / \mathrm{dL})$ & 0.020 \\
Fase $*$ Grupo & Cholesterol $(\mathrm{mg} / \mathrm{dL})$ & 0.361 \\
& Triglyceride $(\mathrm{mg} / \mathrm{dL})$ & 0.012 \\
\hline
\end{tabular}

Fonte: Autores em software estatístico Jamovi (versão 1.6).

\section{Discussão}

Enterococcus faecium 137v - EF137v isolado de queijo de Coalho artesanal tradicionalmente produzido e consumido no estado Pernambuco do nordeste brasileiro foi avaliado quanto às propriedades probióticas anteriormente por Calaça, et al. (2020) e Santos, et al. (2020). Esta propriedade o torna um micro-organismo adequado para ser testado quanto as suas 
habilidades de controle de doenças. O presente estudo foi desenhado para investigar o papel protetor do EF137v para o câncer colorretal, considerando sua capacidade de adesão à parede do intestino e efeito no equilíbrio da microbiota promovendo controle populacional de micro-organismos associados à inflamação crônica e na carcinogênese (Raisch, et al., 2014; Khan, et al., 2017; Geravand, et al., 2019).

Um fato pouco mencionado é a redução de peso durante o câncer colorretal. A composição microbiana intestinal é fortemente influenciada pela dieta, em especial pela administração de probióticos e, por sua vez, influencia a função do intestino (Park \& Bae, 2015). O seu comprometimento acarreta em desequilíbrio metabólico e, para Meyerhardt, et al. (2017) uma questão chave é entender se a perda de peso é um marcador do avanço do câncer ou se influencia o resultado da doença. Urbanska, et al. (2009) comentam que existe uma forte correlação entre dieta e ocorrência do câncer e verificaram em seu estudo que os animais que administraram Lactobacillus acidophilus, mantiveram seu peso corporal estável no período de 17 dias quando comparado aos animais do grupo controle, reduzindo o processo inflamatório no intestino e evitando que adenomas progridam. Para os ratos utilizados em nosso trabalho, nenhuma perda de peso significativa foi observada quando utilizada Enterococcus faecium 137v isolada ou como adjuvante terapêutico da VCR. Não houve declínio na ingestão de alimentos fato que é geralmente associado a condições clínicas importantes como o câncer de intestino.

Kumar, et al. (2012) observaram que uso de Lactobacillus plantarum em animais tratados ocasionou aumento de ganho de peso quando comparado aos animais não tratados. O aumento de peso, relatado pelos autores, pode estar atrelado à uma maior absorção de conteúdos alimentares, aproximadamente 10\%. Asha \& Gayathri (2012) utilizaram Lactobacillus fermentum em animais induzidos ao câncer colorretal e observaram um aumento de $6 \mathrm{~g}$ do peso corporal. $\mathrm{O}$ valor prognóstico da variação de peso relata algumas evidências de que o ganho ou perda de peso durante o câncer têm maior risco de recorrência e morte em comparação quando não há variação de peso (Thivat, et al., 2010). O nosso estudo se caracteriza por uma variação mínima na massa corpórea dos animais tratados seja com EF137V isolado ou o EF137v + VCR, com consumo do probiótico antes e após o crescimento do câncer (Figura 2). No entanto, ainda estão disponíveis dados muito limitados sobre a alteração do peso corporal após a intervenção sobre câncer colorretal e seu impacto no resultado terapêutico (Lee, et al., 2015).

Em nosso estudo foi observado que mesmo os ratos com peso corporal padrão, os níveis de triglicerídeos dos animais tratados GP, G4, G5, G6 e G7 foram significativamente aumentados após indução do câncer quando comparados ao grupo não tratado GN e G3 (Figura 8), enquanto que níveis de colesterol total - CT mantiveram-se estabilizados para todos os grupos. Tal situação pode sugerir alterações do metabolismo devido a presença de adenomas colorretais ou mesmo o estresse pela doença. Dentre as principais alterações metabólicas induzidas pelo câncer colorretal, destacam-se a depleção dos depósitos lipídicos, o incremento da lipólise e a redução da lipogênese e, em consequência, a hiperlipidemia. Esta, por sua vez, pode estar relacionada à inibição da atividade enzimática da lipase lipoprotéica plasmática (Fortes, et al., 2008). Para Yu, et al. (2019) o câncer colorretal pode provocar aumento da permeabilidade intestinal que é acompanhada por inflamação e hiperlipidemia, afetando o fígado, consequentemente. No estudo de Wu, et al. (2017) o consumo de Lactobacillus tem efeitos benéficos na estabilidade de CT, embora não haja alterações óbvias nos níveis séricos de triglicerídeos.

Curiosamente, a hiperlipidemia em animais com câncer colorretal apresenta caquexia associada (Yu, et al., 2019) e, embora os ratos utilizados em nosso estudo tenham apresentado níveis elevados de triglicerídeos após o câncer assim como aumento na motilidade intestinal, a caquexia não foi um fator preponderante. Alguns estudos com modelos de câncer colorretal não relatam em seus experimentos a análise bioquímica para CT e triglicerídeos em ratos com tumores colônicos induzidos por DMH (Asha \& Gayathry, 2012; Kumar, et al., 2012; Desrouillères, et al., 2015). O aumento dos níveis séricos em nosso estudo podem ter relação, por exemplo, com a alteração causada pelo DMH nas enzimas hepáticas que está relacionada ao fígado, que é um órgão importante no metabolismo da maioria dos carcinógenos. Vários carcinógenos ambientais foram relatados como 
causadores de estresse oxidativo hepático durante a tumorigênese extra-hepática (Jaskulski et al. 2020). Portanto, o crescimento do tumor em si pode não causar hiperlipidemia, todavia, não está claro qual evento é o principal responsável pela hiperlipidemia em ratos Wistar. Os estudos que se apresentam na literatura científica ainda são muito conflitantes.

Se o triglicerídeo pode ser responsável pelo desenvolvimento do câncer colorretal sugerido por fornecer combustível para a reoxigenação das células tumorais, indicando um mecanismo de resistência ao longo de regimes de tratamento (Kristen, et al., 2020), o fato de os grupos tratados com o probiótico EF137v isolado (G5 e G6) antes e após a indução do câncer por DMH torna-se intrigante, uma vez que mesmo com níveis alterados de triglicerídeo, a ultrassonografia observou um retardo na progressão do tumor nesses animais, quando comparados ao grupo não tratado com EF137v (GP) e com os grupos que utilizaram a EF137v como adjuvante ao tratamento com a VCR, para promover efeito sinérgico (G4 e G7), como mostra a Figura 6.

De acordo com Asha e Gayathry (2012) ao administrar Lactobacillus fermentum, Lactobacillus plantarum ou VCR houve uma diminuição na ocorrência de focos de criptas aberrantes no cólon, no entanto, ao combinar o tratamento, níveis de inibição de $90 \%$ eram alcançados, o que comprova possível efeito sinérgico, diferente do ocorrido em nossos experimentos. Segundo Chen, et al. (2012) ao administrar por via oral Lactobacillus acidophilus foi possível reduzir a progressão do câncer colorretal e as lesões em tecidos adjacentes. Levando a acreditar que o consumo prévio do L. acidophilus pode suprimir a evolução tumoral, de acordo com o observado no grupo G5 em que a administração do EF137v antecedeu seis semanas à indução do câncer. Para Kumar, et al. (2012), que utilizaram L. plantarum na diminuição do câncer colorretal, administrar probióticos por um período longo é importante para alcançar melhores efeitos de inibição.

Talvez você deve estar se perguntando sobre o papel da ultrassonografia em nossas análises uma vez que foge ao padrão de estudos comumente relacionados ao acompanhamento do câncer de cólonl em animais e em humanos. Uma das principais razões é o modo de rastreamento do câncer de cólon seguir um protocolo estabelecido em que recomenda-se, a partir dos 50 anos de idade (para aqueles que não tem histórico familiar) e 40 anos de idade (para histórico deste tipo de câncer na família) os procedimentos de início de triagem pelo exame de colonoscopia, um exame invasivo, caro e que necessita de sedação, cuja indicação deve ser baseada em sinais, sintomas e fatores de risco (Rex, et al., 2017; INCA, 2020). Especula-se, no entanto, o aumento na ocorrência de câncer de cólon em jovens entre 18-30 anos de idade que se deve, em parte, ao viés de detecção tardia (Swati, et al., 2020). Neste caso, o valor clínico do rastreamento é refletido em sua capacidade de prevenir a morbidade, mortalidade e custo excessivo do tratamento do câncer, detectando lesões significativas antes que se tornem cancerosas e um câncer em estágio inicial antes de se espalhar além da parede intestinal (Simon, 2016).

Do ponto de vista teórico, várias combinações de técnicas de imagem in vivo não invasivas podem ser usadas para avaliar a evolução das características do câncer, entre elas a ultrassonografia. As medições de parâmetro único não refletem a heterogeneidade tumoral, e uma análise combinada de diferentes parâmetros seria esperada para fornecer informações complementares para a previsão da resposta do tumor, prognóstico e caracterização biológica do câncer colorretal (GarcíaFigueiras et al. 2018). A análise por ultrassonografia em nosso estudo, identificou parâmetros morfológicos de parede intestinal diferenciados após iniciação do câncer colorretal em ratos Wistar, colocando a ultrassonografia em sugestão como um exame adicional de fácil acesso, pouco invasivo e barato para rastreamento do câncer em indivíduos jovens que podem submeter a ultrassonografia abdominal aos exames de rotina semestrais ou anuais.

\section{Conclusão}

O EF137v mostrou-se forte candidato como agente de proteção para indivíduos saudáveis que apresentam fatores genéticos de promoção ao câncer, bem como a prevenção da doença para vários perfis de indivíduos e àqueles diagnosticados que buscam por adjuvantes capazes de atenuar os efeitos colaterais dos quimioterápicos e o retardo na progressão do câncer 
colorretal, melhorando a sobrevida. Um grande diferencial do nosso estudo está em adicionar a ultrassonografia abdominal para o acompanhamento dos estudos experimentais com animais submetidos à indução de tumor, otimizando as análises e reduzindo o número de eutanásias necessárias para o acompanhamento, em tempo real, sobre o desenvolvimento da doença, sem a necessidade de anestésicos ou cirurgias, situação que reduz o estresse dos animais e qualifica os resultados dos procedimentos adotados.

Estudos adicionais como avaliação histopatológica e imunohistoquímica de intestino e fígado dos animais testados estão sendo realizados a fim de averiguar os efeitos do probiótico de forma sistêmica e integral.

\section{Agradecimentos}

À Coordenação de Aperfeiçoamento de Pessoal de Nível Superior (CAPES).

\section{Referências}

Asha \& Gayathri D. (2012). Synergistic impact of Lactobacillus fermentum, Lactobacillus plantarum and vincristine on 1,2-dimethylhydrazine-induced colorectal carcinogenesis in mice. Exp. Ther. Med. 3:1049-1054.

Desrouillères, K., Millette, M., Vu, K. D., Touja, R. \& Lacroix M. (2015). Cancer preventive effects of a specific probiotic fermented milk containing Lactobacillus acidophilus CL1285, L. casei LBC80R and L. rhamnosus CLR2 on male F344 rats treated with 1,2-dimethylhydrazine. J. Funct. Foods, 17:816827.

Fortes, R. C. (2008). Alterações lipídicas em pacientes com câncer colorretal em fase pós-operatória: ensaio clínico randomizado e duplo-cego com fungos Agaricus sylvaticus. Rev bras. colo-proctol., 28(3):281-288.

Gamallat, Y., Meyiah, A., Kuugbee, E. D., Hago, A. M., Chiwala, G., Awadasseid, A., Amba, D., Zhang, X., Shang, X., Luo, F. \& Xin, Y. (2016). Lactobacillus rhamnosus induced epithelial cell apoptosis, ameliorates inflammation and prevents colon cancer development in an animal model. Biomed Pharmacother. 83:536-541.

García-Figueiras, R., Baleato-González, S., Padhani, A. R., Luna-Alcalá, A., Marhuenda, A., Vilanova, J. C., Osorio-Vázquez, I., Martínez-de-Alegría, A. \& Gómez-Caamaño, A. (2018). Advanced Imaging Techniques in Evaluation of Colorectal Cancer. Radiographics. 38(3):740-765.

Hochman, B., Nahas, F. X., Filho, R. S. O. \& Ferreira, L. M. (2005). Desenhos de pesquisa. Acta Cirúsgica Brasileira. $20(2): 1-8$.

Hradicka, P., Beal, J., Kassayova, M., Foey, A. \& Demeckova, V. (2020). A Novel Lactic Acid Bacteria Mixture: Macrophage-Targeted Prophylactic Intervention in Colourectal Cancer Management. Microorganisms, 8:387.

INCA (2020). Estimativa 2020: Incidência de Câncer no Brasil. Instituto Nacional de Câncer.

Itiane, B. J., Julia, U., Fernanda, B., Fernanda, M., Taiciane, G., Natalie, G. C., Flávio, C., Fabiane, B. G., Ângela, M. F., Wladimir, P. S., Robson, A. \& Simone, P. (2020). In vivo action of Lactococcus lactis subsp. lactis isolate (R7) with probiotic potential in the stabilization of cancer cells in the colorectal epithelium, Process Biochemistry, 91:165-171.

Raisch, J., Buc, E., Bonnet, M., Sauvanet, P., Vazeille, E., Vallee, A., Echelotte, P., Darcha, D., Pezet, D., Bonnet, R.,Bringer, M. A. \& Darfeuille-Michaud, A. (2014). Escherichia coli B2 associada ao câncer de cólon coloniza a mucosa intestinal e promove a proliferação celular. World J. Gastroenterol., 20:65606572 .

Kristen, D., Brantley, A., Riis, H., Rune, E., Ole, T., Holger, J. M. \& Timothy, L. (2020). The association of serum lipid levels with colorectal cancer recurrence. Cancer Epidemiology, 66.

Kumar, R. S., Kunnami, P., Yuvaraj, N., Paari, K. A., Pattukumar, V., Thirunavukkarasu, C. \& Arul, V. (2012). Lactobacillus plantarum AS1 Isolated from South Indian. Appl. Biochem. Biotechnol., 166:620-631.

Lan, J., Sun, L., Xu, F., Liu, L., Hu, F., Song, D., Hou, Z., Wu, W., Luo, X., Wang, J., Yuan, X., Hu, J. \& Wang, G. (2019). M2 Macrophage-Derived Exosomes Promote Cell Migration and Invasion in Colon Cancer. Cancer Res. 79(1):146-158.

Lee, D. W., Han, S. W., Cha, Y., Lee, K. H., Kim, T.Y., Oh, D., Im, S. A., Bang, Y. J., Park, J. W., Ryoo, S. B., Jeong, S. Y., Kang, G. H., Park, K. J. \& Kim, T. Y. (2015). Prognostic influence of body mass index and body weight gain during adjuvant FOLFOX chemotherapy in Korean colorectal cancer patients. BMC Cancer., 15:690.

Lenoir, M., Del Carmen, S., Cortes-Perez, N. G., Lozano-Ojalvo, D., Muñoz-Provencio, D., Chain, F., Langella, P., LeBlanc, A., LeBlanc, J. G. \& Bermúdezumarán, L. G. (2016). Lactobacillus casei BL23 regulates Treg and Th17 T-cell populations and reduces DMH-associated colorectal cancer. J Gastroenterol., 51(9):862-73.

Meyerhardt, J. A., Kroenke, C. H., Prado, C. M., Kwan, M. L., Castillo, A., Weltzien, E., Cespedes Feliciano, E. M., Xiao, J. \& Caan, B. J. (2017). Association of Weight Change after Colorectal Cancer Diagnosis and Outcomes in the Kaiser Permanente Northern California Population. Cancer Epidemiol Biomarkers Prev., 26(1):30-37.

Park, S. \& Bae, J. H. (2015). Probiotics for weight loss: a systematic review and meta-analysis. Nutr Res. 35(7):566-75. 
Research, Society and Development, v. 10, n. 6, e9110615354, 2021

(CC BY 4.0) | ISSN 2525-3409 | DOI: http://dx.doi.org/10.33448/rsd-v10i6.15354

Sharma, P., Kaur, S., Kaur, R., Kaur, M. \& Kaur, S. (2018). Proteinaceous Secretory Metabolites of Probiotic Human Commensal Enterococcus hirae 20c, E. faecium 12a and L12b as Antiproliferative Agents Against Cancer Cell Lines. Front Microbiol., 15,9:948.

Swati, G., Patel, C. \& Richard, B. (2020). Colorectal Cancer in Persons Under Age 50: Seeking Causes and Solutions, Gastrointestinal Endoscopy Clinics of North America.,30(3):441-455.

Thirabunyanon, M. \& Hongwittayakorn, P. (2013). Potential probiotic lactic acid bacteria of human origin induce antiproliferation of colon cancer cells via synergic actions in adhesion to cancer cells and short-chain fatty acid bioproduction. Appl Biochem Biotechnol. 169(2):511-25.

Thirabunyanon, M., Boonprasom, P. \& Niamsup, P. (2009). Probiótico potencial de bactérias de ácido láctico isoladas de leites lácteos fermentados em antiproliferação de células de câncer de cólon. Biotechnol Lett., 31:571-576.

Thivat, E., Thérondel, S., Lapirot, O., Abrial, C., Gimbergues, P., Gadéa, E., Planchat, E., Kwiatkowski, F., Mouret-Reynier, M. A., Chollet, P. \& Durando, X. (2010). Weight change during chemotherapy changes the prognosis in non metastatic breast cancer for the worse. BMC Cancer. 25:10:648.

Barton, T. P., Cruse, J. P. \& Lewin, M. R. (2008) Alterações nos lipídios séricos relacionados à presença de câncer de cólon experimental. Br. J. Câncer., $56: 451-454$.

Urbanska, A. M., Bhathena, J., Martoni, C. \& Prakash, S. (2009). Estimation of the Potential Antitumor Activity of Microencapsulated Lactobacillus acidophilus Yogurt Formulation in the Attenuation of Tumorigenesis in Apc(Min/+) Mice. Dig. Dis. Sci. 54:264-273.

Wu, Y., Zhang, Q., Ren, Y. \& Ruan, Z. (2017). Effect of probiotic Lactobacillus on lipid profile: A systematic review and meta-analysis of randomized, controlled trials. PLoS One. 8:12(6):e0178868. 